\title{
Energy in General Relativity - the case of the neutron star
}

\section{Wallis M.K., Marshall T.W.}

Buckingham Centre for Astrobiology, University of Buckingham MK18 1EG, UK;

E-mail: Marshall<maxkwallis@gmail.com>;

The current widely accepted theory of gravitational collapse relies on an assumption - the attractive gravity paradigm - used in selecting the boundary condition at the centre. This assumption is basic to the Newtonian theory, but it is not appropriate in General Relativity. We have integrated the field equations of the latter for a sphero-symmetric neutron star in the static limit, modelling the stellar material as a free Fermi gas, and following the treatment of Tolman, Oppenheimer and Volkoff. If the outer surface is at a radius exceeding the gravitational radius $2 M G / c^{2}$, we found for $M$ exceeding the critical mass, there exist solutions with the stellar material entirely confined to a shell, whose inner radius also exceeds that radius.

We now address the question of how the repulsion of stellar material away from the centre is produced. We proceed from the energy pseudotensor of Landau and Lifshitz, modifying it along the lines suggested by Logunov and Mestvirishvili so that it becomes a tensor. This procedure requires the adoption of a preferred system of coordinates, whereby the radius appearing in the well-known Schwarzschild free-space metric is transformed to the harmonic radius. The gravitational energy then becomes a well-defined function of position, taking negative values inside the neutron shell, so that the field associated with it is repulsive. The equality of gravitational and inertial mass is guaranteed, while the Equivalence Principle holds only in its weaker form.

Keywords: neutron star, Oppenheimer-Volkoff, energy tensor, repulsive gravity.

DOI: $10.18698 / 2309-7604-2015-1-546-558$

\section{Introduction}

It is well known that, in General Relativity (GR), it is not possible to define a covariant energy tensor, and the early history of GR is largely dominated by the frustrated efforts of the theory's founders to define such a tensor.

In 1913 Einstein[1] had written

The tensor of the gravitational field energy, together with the tensor of the material energy, is the source of the gravitational field. The gravitational field energy occupying a special position as compared with all other forms of energy would have inadmissible consequences.

This position he abandoned after adopting the geometrical interpretation of GR in 1915, because the strong form of the Equivalence Principle, on which the geometrical interpretation is based, does not allow the existence of any favoured coordinate frame; without such a frame it is not possible to construct an energy tensor as was established by Hilbert[2] two years later. Einstein nevertheless reverted to his 1913 position in 1918, when he derived the quadrupole formula[3] for the emission of gravitational waves from a bounded non-spherical source; there he was obliged to 
use a specific frame which later came to be called (Fock[4]) the harmonic coordinates. He had already changed his mind twice about gravitational waves when he wrote that article. Throughout the subsequent four decades, not only would he change twice more, but his oscillations of opinion were mirrored in the communal understanding of gravity, causing confusion among virtually all of the leading scholars in the field [5]. The communal view changed radically when observation of the Hulse-Taylor pulsar[6] confirmed that this binary system is losing energy at the rate predicted by the quadrupole formula, and subsequent measurement of its changing keplerian orbit parameters over decades have confirmed the harmonic-frame predictions to high accuracy.

Einstein attempted also to establish the equality of inertial and gravitational mass in a second article of 1918[7], but, because he wanted to stay within the geometrical interpretation, he again had to use a pseudotensor for the energy. However, it was established that this result also is frame dependent by Logunov and Mestvirishvili[8], who argued that harmonic coordinates are necessary to describe properly the energy of gravitational compression in objects like white dwarfs and neutron stars. The argument in the latter work was strongly influenced by the earlier work of Fock [4], but also they moved further than Fock did in their preference for the harmonic frame. Following a suggestion by Rosen[9], they argued that, in addition to the Riemann metric of GR, there is an underlying flat space with the Minkowski metric of Special Relativity. Certain field quantities related to the Riemann metric have to satisfy equations additional to the geometrical ones. These are expressed as covariant derivatives in the Minkowski space, but in the unique harmonic coordinates the covariant derivatives become ordinary derivatives, that is they do not involve derivatives of the metric coefficients.

Such an extension of GR enables us to convert the energy pseudotensor of Landau and Lifshitz[10] into a tensor, or more simply as suggested in [8], to keep using the pseudotensor but with the harmonic radius.

The present article applies the above ideas to neutron stars, and in particular to solutions we have obtained to the Oppenheimer-Volkoff equations[11] for masses at which these authors considered a solution to be impossible. We show that the breakdown of their solution for high masses resulted from their carrying over from Newtonian gravity the assumption that the pressure increases monotonically towards the centre of a neutron star. We then show that, if we integrate from the surface inwards, rather than from the centre outwards, with no assumption other than that the outer surface lies beyond the gravitational radius $2 G M / c^{2}$, where $M$ is the gravitational mass, then the stellar material is entirely confined to a shell whose inner radius also exceeds that radius. Such solutions indicate that supermassive neutron stars, and even supermassive white giants with 
electrons rather than neutrons supplying the internal pressures, may be present within our own galaxy, with Sagittarius $A^{*}$ at the galactic centre as a prime candidate. In supermassive objects the shell becomes very thin, and we have likened them to a giant football; for example the radius of Sagittarius $A^{*}$ is 13 million km, but its shell has a thickness of less than $10,000 \mathrm{~km}$.

There must be a force acting in the "empty" interior region to compress the stellar material into such a shell; Newtonian attractive gravity is replaced by GR repulsive gravity. Geometrical GR, based on the strong Equivalence Principle, pretends to have abolished gravitational force, but now that we have an energy tensor we may reinstate it. Only the Weak Equivalence Principle (WEP) remains, which states that the mass associated with all forms of energy (including gravitational) gravitate equally and the total inertial mass (defined below) of an isolated object is equal to its gravitational mass.

\section{The neutron star}

Oppenheimer and Volkoff (OV)[11] took the metric outside and inside a neutron star to be

$$
d s^{2}=B(r) d t^{2}-A(r) d r^{2}-r^{2}\left(d \theta^{2}+\sin ^{2} \theta d \phi^{2}\right)
$$

We introduce the variables $u(r)$ and $v(r)$, defined by

$$
A=\frac{r}{r-2 u}, B=\frac{v^{2}}{A} \text {. }
$$

Then the OV field equations become, in the same units as used there ( $G=c=1$, mass unit=9.2625 $\mathrm{M}_{\text {sun }}$,

$$
\begin{aligned}
& \frac{d u}{d r}=4 \pi r^{2} \rho, \\
& \frac{d v}{d r}=\frac{4 \pi r^{2}(p+\rho) v}{r-2 u}, \\
& \frac{d p}{d r}=-\frac{(p+\rho)\left(\mathrm{u}+4 \pi \mathrm{r}^{3} p\right)}{r(r-2 u)} .
\end{aligned}
$$


The density $\rho(r)$ and pressure $p(r)$ were taken to satisfy the ideal Fermi gas equation of state

$$
\begin{aligned}
& 4 \pi \rho=4(1+2 \theta) \sqrt{\theta(1+\theta)}-4 t \\
& 4 \pi \rho=\frac{4}{3}(2 \theta-3) \sqrt{\theta(1+\theta)}+4 \\
& t=\ln (\sqrt{\theta}+\sqrt{1+\theta}) .
\end{aligned}
$$

Note that the OV parameter $t$ is related to our $\theta$ by $\theta=\sinh ^{2} t$. Now we put $\theta=0, \quad u=M$, $v=1$ in an exterior region $r>R$, where $R$ is an arbitrarily chosen radius greater than the gravitational radius $2 M$. For $r$ less than $R$ we solve (3) with the third field equation written as

$$
\frac{d \theta}{d r}=-\frac{2(1+\theta)\left(u+4 \pi r^{3} p\right)}{r(r-2 u)},
$$

and continuous boundary values at $r=R$. There results a solution, having a zero of $\theta$ at a certain value $r=R_{1}$ and with $u_{1}=u\left(R_{1}\right)$ negative and $v_{1}=v\left(R_{1}\right)$ positive. We then take $\theta=0, u=u_{1}, v=$ $v_{1}$ in the interior region $r<R_{1}$. Since $\rho$ is a multiple of $\theta^{3 / 2}$ for small $\theta, \rho(r)$ is continuously differentiable at both $r=R$ and $r=R_{1}$. So our solution of the OV equation is a shell-like structure with the massive particles all occupying the space between $r=R_{1}$ and $r=R$. 


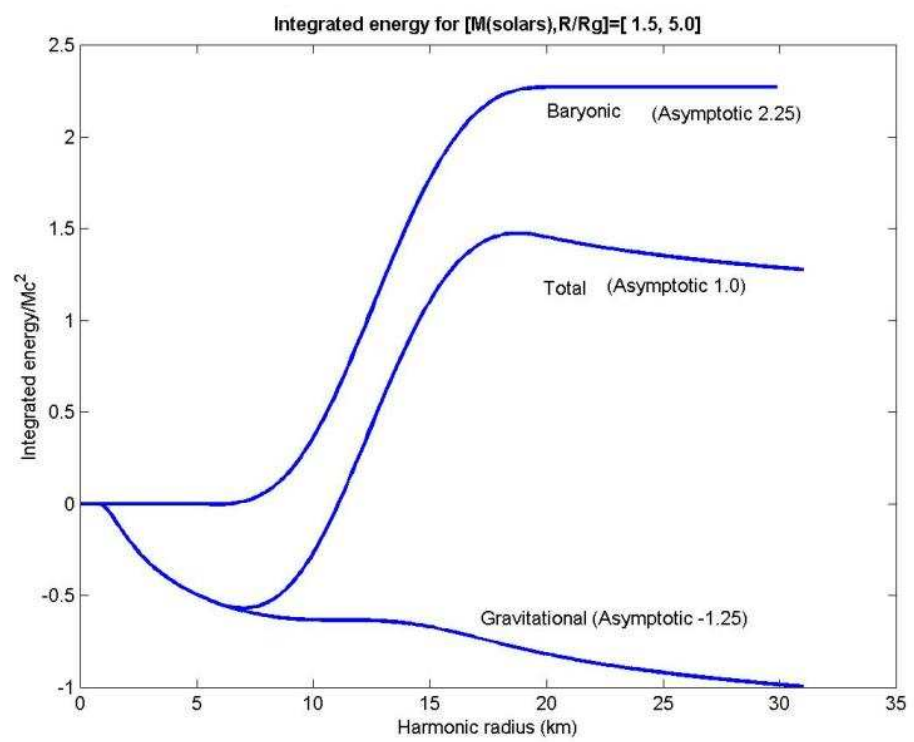

Fig. 1. Plots of integrated energy of a Fermi gas $(\mathrm{T}=0)$ neutron star with mass $\mathrm{M}=1.5 \mathrm{Mo}$, whose baryonic mass exceeds the Tolman-Oppenheimer-Volkoff limit of $\sim 2$ Mo. The radius in the Schwarzschild frame was taken as $\mathrm{R}=5 \mathrm{Rg}$ and the maximum baryonic density is $219 \mathrm{Mt} / \mathrm{cm}^{3}$. The profiles show the baryonic mass density dropping to zero within the gravitational radius $(4.5 \mathrm{~km})$, but the gravitational energy density significant. The gravitational energy is low within the shell $(10-15 \mathrm{~km})$ but extends far beyond it.

The theory gives us no indication how to determine the relation between $R$ and $M$; the arbitrary nature of the solution arises because, as a consequence of the OV equation of state (4), the neutron density is strictly zero inside the shell, so there is no boundary condition at $r=0$, which is a contrast with the $\mathrm{OV}$ theory, based on an incorrect boundary condition. For the value $M=1.5 M_{\odot}$ we have put $R$ equal to 5 times the gravitational radius $2 G M / c^{2}$, which corresponds to a typical neutron star with $R=22.1 \mathrm{~km}$; this gives $R_{1}$ around $10 \mathrm{~km}$, well outside the gravitational radius of $4.5 \mathrm{~km}$. Both of these radii will be slightly amended after changing to the harmonic coordinates treated in the next section. From computed results in terms of $\rho(r)$, we extract two pieces of data, namely the maximum baryonic density and the baryonic mass, defined as the integral of $\rho$ between $r=R_{1}$ and $r=R$. In this case the maximum density is 219 megatonnes $/ \mathrm{cm}^{3}$, which is comfortably less than the limit at which hyperons may be produced, and the baryonic mass is about 2.25M (Fig.1). The difference between the latter and $M$, called the mass defect, may be ascribed to the negative gravitational energy, whose contribution we consider in the following sections. 
The theory also predicts that neutron stars far in excess of the OV limit can exist; we have calculated the values of $R_{1}$, as well as the maximum density and baryonic mass for a range of $M$ from 1.5 to $10^{5}$ solar masses, and with $R$ somewhat greater than the gravitational radius $2 G M / c^{2}$. A striking feature emerges, namely as $M$ goes into the supermassive range $R_{1}$ can approach very close to $R$, that is the density profile describes an object whose baryonic mass is concentrated in a very thin shell; we have called this object a football collapsar (Marshall and Wallis [24]). Because of the rapid enlargement of the surface area as $M$ increases, the maximum density within the shell decreases steadily even though the shell width is decreasing, so that somewhere around $M=10^{5} M_{\odot}$ a large proportion of neutrons decay through the weak interaction and the neutron star becomes a supermassive white giant, in which the pressure supporting it is provided by electrons as in white dwarfs. The mass defect increases steadily with $M$.

\section{The harmonic condition}

Field theories of gravity, beginning with [3], impose the non-covariant condition

$$
\partial_{\mu} \Phi^{\mu v}=0
$$

where the field quantity $\Phi^{\mu v}$ is related to the Riemann metric by

$$
\Phi^{\mu \nu}=g^{\mu v} \sqrt{ }-g
$$

Einstein imposed this condition in order to establish the existence of gravitational waves, which prompted the famous criticism from Eddington that such waves "travel at the speed of thought"[12] thereby initiating a fifty-year debate on the subject[5]. Our proposal, referred to in the previous section, is to meet Eddington's criticism by making the condition covariant, that is

$$
D_{\mu} \Phi^{\mu v}=0, \Phi^{\mu v}=g^{\mu v} \sqrt{\frac{g}{\gamma}}
$$

where $D_{\mu}$ is the covariant Minkowski derivative associated with the metric $\gamma_{\mu v}$, the special case being that, when $\gamma_{\mu \nu}$ is the galilean metric 


$$
d \sigma^{2}=d t^{2}-d x^{2}-d y^{2}-d z^{2}
$$

so that the covariant derivative becomes an ordinary partial derivative and $\gamma=-1, g_{\mu \nu}$ satisfies the harmonic condition (6). In the case of the static sphero-symmetric Riemann metric (2), the harmonic coordinates are $(t, x, y, z)=\left(t, r_{H} \sin \theta \cos \varphi, r_{H} \sin \theta \sin \varphi, r_{H} \cos \theta\right)$ where $r_{H}$ satisfies (see Fock[4], also Weinberg[13] (8.1.15))

$$
\frac{d}{d r}\left(r^{2} \sqrt{\frac{B}{A}} \frac{d r_{H}}{d r}\right)=2 r_{H} \sqrt{A B}
$$

In terms of our variables $u$ and $v$, this becomes

$$
\begin{gathered}
\frac{d^{2} r_{H}}{d r^{2}}+\left[\frac{1}{r}+\frac{1-2 u^{\prime}}{r-2 u}+\frac{v^{\prime}}{v}\right] \frac{d r_{H}}{d r}=\frac{2}{r(r-2 u)} r_{H}, \\
\text { that is } \frac{d^{2} r_{H}}{d r^{2}}+\left[\frac{1}{r}+\frac{1+4 \pi r^{2}(p-\rho)}{r-2 u}\right] \frac{d r_{H}}{d r}=\frac{2}{r(r-2 u)} r_{H} .
\end{gathered}
$$

This has to be solved with appropriate boundary conditions. In the regions $r>R$ and $r<$ $R_{1}$, where $p$ and $\rho$ are zero, the general solution is

$$
r_{H}=a(r-u)+b\left[u+\frac{r-u}{2} \ln \left(1-\frac{2 u}{r}\right)\right]
$$

and, with $u=M$ in $r>R$ and $u=u_{1}$ in $r<R_{1}$, these may be matched, with suitable $a$ and $b$, to a numerical solution in the shell region $R_{1}<r<R$. Any nonzero value of $b$ at $r=0$ is unphysical, so therefore putting $a=1$ in the exterior region and $b=0$ in the interior region fixes a unique value $b=b_{0}(M, R)$ in the exterior region and $a=a_{1}(M, R)$ in the interior region. On carrying out a numerical integration for values of $M$ ranging from 1.5 to $10^{5}$ solar masses, we found consistently monotonic increasing $r_{H}(r)$ with $r_{H}$ taking a positive value $r_{H 0}$ at $r=0$. Note that, for $r \rightarrow \infty$,

$$
r_{H} \sim \mathrm{r}-\mathrm{M}+\left(\frac{b_{0} M^{3}}{3 r^{2}}\right)+O\left(\frac{M^{4}}{r^{3}}\right)
$$


After transforming from $r$ to $r_{H}$, the Riemann metric becomes

$$
d s^{2}=\frac{v^{2}(r-2 u)}{r} d t^{2}\left[\frac{r}{r-2 u}\left(\frac{d r}{d r_{H}}\right)^{2} \frac{x_{i}^{H} x_{j}^{H}}{r_{H}^{2}}+\frac{r^{2}}{r_{H}^{2}}\left(\delta_{i j}-\frac{x_{i}^{H} x_{j}^{H}}{r_{H}^{2}}\right)\right] d x_{i}^{H} d x_{j}^{H}
$$

and the Minkowski metric becomes

$$
d \sigma^{2}=d t^{2}-\delta i j d x^{H} i d x^{H} j,
$$

or, if we wish to return to the Schwarzschild coordinates,

$$
d \sigma^{2}=d t^{2}-\left(\frac{d r_{H}}{d r}\right)^{2} d r^{2}-r_{H}^{2}\left(d \theta^{2}+\sin ^{2} \theta d \phi^{2}\right)
$$

\section{The energy tensor}

The covariant form of the Landau-Lifshitz pseudotensor $t^{\mu v}{ }_{g}$ (see [10] page $280^{4}$ ) combines with the material stress tensor,

$$
T^{\mu v}=(p+\rho) \frac{d x^{\mu}}{d s} \frac{d x^{v}}{d s}-p g^{\mu v}
$$

to give a total energy tensor

$$
t^{\mu v}=t_{g}^{\mu v}+T^{\mu v}=\frac{\gamma}{16 \pi g} D_{\lambda} D_{k}\left(\Phi^{\mu v} \Phi^{\lambda k}-\Phi^{\mu \lambda} \Phi^{v k}\right),
$$

which satisfies, in the harmonic frame, the conservation equation

$$
\partial_{\mu}\left[(-g) t^{\mu \nu}\right]=0
$$

4 Their pseudotensor is a bilinear form in the first derivatives of $\Phi^{\alpha \beta}$. To convert it into a tensor, all such derivatives are replaced by Minkowski covariant derivatives. 
and enables us to define the covariant 4-momentum

$$
P^{\mu}={ }_{o}(-g) t^{\mu 0} d V
$$

The harmonic metric (15) gives [14][15]

$$
\Phi^{00} \frac{r^{3}}{v r_{H}^{2}(r-2 u)} \frac{d r}{d r_{H}}, \Phi^{0 i}=\Phi^{i 0}=0
$$

and

$$
\Phi^{i j}=-\Phi_{1}\left(\mathrm{r}_{H}\right) \frac{X_{i}^{H} X_{j}^{H}}{r_{H}^{2}}-\Phi_{2}\left(\mathrm{r}_{H}\right)\left(\delta_{i j}-\frac{X_{i}^{H} X_{j}^{H}}{r_{H}^{2}}\right)
$$

Where

$$
\Phi_{1}=\frac{v r(r-2 u)}{r_{H}^{2}} \frac{d r_{H}}{d r}, \Phi_{2}=v \frac{d r}{d r_{H}}
$$

So, in harmonic coordinates, $-16 \pi g t^{00}$ simplifies to

$$
-16 \pi g t^{00}=\partial_{i}^{H}\left(\Phi^{i j} \frac{x_{j}^{H}}{r_{H}} \frac{d \Phi^{00}}{d r_{H}}\right)=\partial_{i}^{H}\left(\frac{x_{i}^{H}}{r_{H}} \Phi_{1} \frac{d \Phi^{00}}{d r_{H}}\right),
$$

This is a cartesian divergence, and its integral over the region $r_{H}<r_{1}$ gives the total energy within it, namely

$$
P^{0}\left(r_{1}\right)=\int_{r_{H}<r_{1}}(-g) t^{00} d V=\frac{r_{1}^{2}}{4}\left[\Phi_{1} \frac{d \Phi^{00}}{d r_{H}}\right]_{r_{H}=r_{1}}
$$

that is 


$$
P^{0}\left(r_{1}\right)=\int_{r_{H}<r_{1}}\left[-\frac{r^{3}}{r_{H}^{2}} \frac{d r}{d r_{H}}+\frac{r^{3}}{2 r_{H}(r-2 u)}\left(\frac{d r}{d r_{H}}\right)^{2}+\frac{r^{4}}{2 r_{H}^{3}}\right]_{r_{H}=r_{1}} .
$$

In the latter derivation the behaviour of $r_{H}$ as $r$ tends to zero, commented on in the previous section, ensures that the energy density is nonsingular there. For large $r_{1}$ the integrated energy becomes, in view of the asymptotic behaviour (14),

$$
P^{0}\left(r_{1}\right)=M+\frac{7 M^{2}}{2 r_{1}}+O\left(\frac{M^{3}}{r_{1}^{2}}\right)
$$

in agreement with expressions previously obtained by Marshall[14] and by Gershtein, Logunov and Mestvirishvili[15]. The asymptotic behaviour of $P^{0}$ for large $r_{1}$ confirms that the inertial and gravitational masses are equal, that is Weak Equivalence is satisfied.

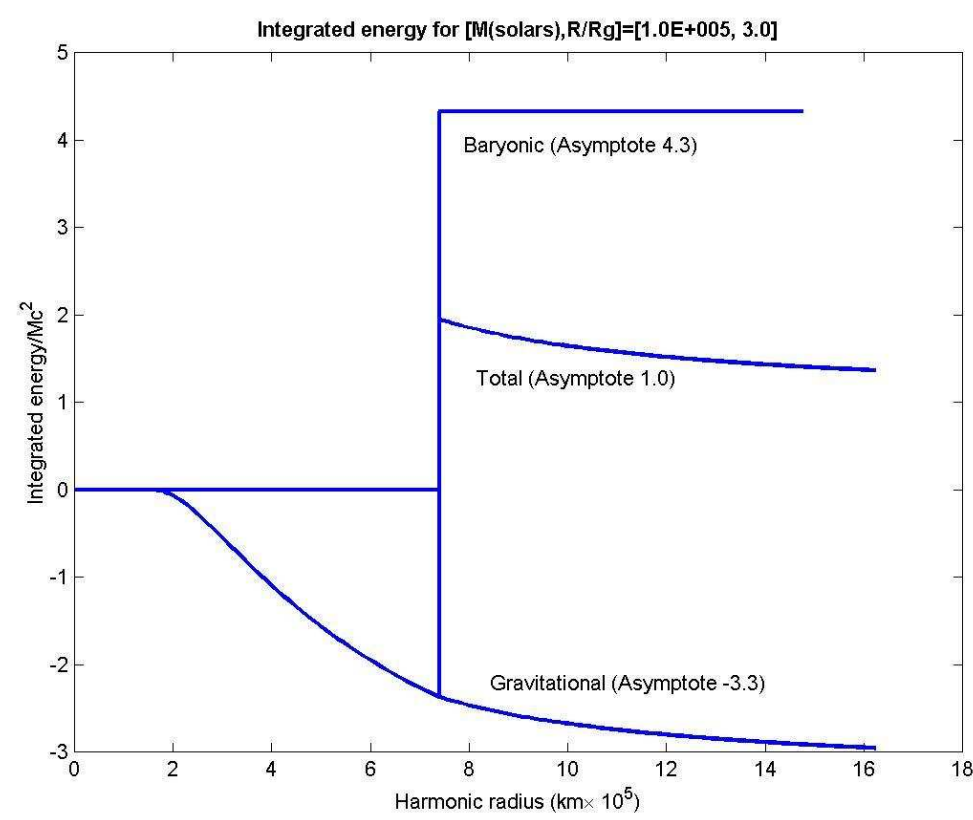

Fig. 2. Plots like Fig.1 but for a neutron star of $10^{5} \mathrm{Mo}$. The baryonic shell is much narrower, appearing discontinuous on this scale, but with peak density far lower than the 1.5Mo case. The radius in the Schwarzschild frame was taken as $\mathrm{R}=3 \mathrm{Rg}$. The 'hole' within $2 \mathrm{~km}$ is an artefact of the zero-T neutron Fermi gas. The mass defect, corresponding to the high central concentration of negative gravitational energy, is the asymptotic value 3.3. 
In Figures 1, 2 we plot $P^{0}(r)$ against $r_{H}(r)$ for the gravitational masses $M=1.5 M_{\odot}$ and 10 ${ }^{5} M_{\odot}$. Note the resemblance of the first plot to the "artist's impression" of Marshall[14], in which we sketched the likely distribution of the total energy inside the collapsar without the benefit of the energy expression derived here. The second Figure exhibits similar behaviour, but, owing to the extremely small shell width in which the neutron content is confined, the smooth curve becomes much more abrupt. Note that the neutron or "baryonic" contribution to $P^{0}$, in accordance with the conservation equation (20), is now defined as the integral of

$$
-g T^{00}=-g \rho g^{00}=\frac{\rho r^{5}}{r_{H}^{4}(r-2 u)}\left(\frac{d r}{d r_{H}}\right)^{2}
$$

This is different from the baryonic mass defined in Section 2, and gives somewhat higher values than we obtained there for the mass defect, that is the value times -1 of the gravitational energy at $r=\infty$. As commented in Section 2, Figure 2 shows a very large decrease in the density maximum as compared with Figure 1.

\section{Conclusion}

The modification of geometrical GR to convert it into a field theory has a history going back to before GR; some of that history was recounted in [5]. The observational evidence for gravitational waves emitted by pulsars should have provoked a lot more reappraisal of the situation, but sadly there has been very little. One outstanding exception, aside from those[8] already quoted, is Weinberg ([13], preface and section 9.9).

In the context of gravitational waves, the relevance of the energy tensor, as opposed to a pseudotensor, is especially significant. We note that even Fock [4], who did so much to emphasize the importance of the harmonic coordinates, was sufficiently opposed to a flat-space version of GR to reject the idea of an energy tensor, and consequently claimed that the field energy could not be localized. The direct observation a passing of a gravitational wave, for example in the LIGO project[16], would surely make us reverse Fock's judgement.

Our findings add further support to the notion of a localized gravitational energy, in particular through allowing us to distinguish the baryonic from the gravitational mass $M$; the large value of the negative gravita-tional energy in the supermassive range explains why the neutrons are compressed into such a narrow shell. 
There is an obvious part of our theory remaining to be completed, namely the relation between $M$ and the external radius $R$. As commented in section 2, this completion requires us to use a less idealized equation of state for the neutron "gas". There are a vast array of possible candidates for such an extension; the simplest would be to treat it as a perfect Fermi gas with nonzero temperature, but more realistically a model including neutron-neutron interaction would be preferred. We made a preliminary attempt in the first direction earlier[18], but without using the energy tensor or the harmonic coordinates.

\section{References}

1. Einstein A. (1913). Naturforsch. Gesellschaft Zurich, 58, 284-290.

2. Hilbert D. (1917). Gott. Nachr. 4, 21

3. A. Einstein A (1918). Sitzungsber. preuss. Akad. Wiss, 1, 154-167.

4. Fock V.A. (1966). Space, Time and Gravitation. Oxford: Pergamon.

5. Kennefick D. (2007). Traveling at the Speed of Thought. Princeton: Princeton Univ. Press.

6. Hulse R.A., Taylor J.H. (1975). Astrophys. J., 195, 51-53.

7. Einstein A. (1918). Sitzungsber. preuss. Akad. Wiss., 1, 448.

8. Logunov A.A., Mestvirishvili M.A. (1989). The Relativistic Theory of Gravitation. Moscow: MIR [World].

9. Rosen N. (1940). Phys. Ref., 57, 147, 150.

10. Landau L.D., Lifshitz E.M. (1975). The Classical Theory of Fields. Oxford:ButterworthHeinemann.

11. Oppenheimer J.R., Volkoff G.M. (1939). Phys. Rev., 54, 540.

12. Eddington A.S. (1924). The Mathematical Theory of Relativity. Cambridge: University Press.

13. Weinberg S. (1972). Gravitation and Cosmology. New York: John Wiley.

14. Marshall T.W. (2011). Fields tell matter how to move. arXiv, abs/1103.6168.

15. Gershtein S.S., Logunov A.A., Mestvirishvili M.A. (2014). The effect of accumulation of excess energy of a body in gravitational compression. arXiv, abs/1404.3687.

16. Report on the LIGO project, Retrieved from http://www.ligo-la.caltech.edu/LLO/ overviewsci.htm 
Proceedings of International Conference PIRT-2015

17. Marshall T.W., Wallis M.K. (2010). J. Cosmology, 6, 1473-1484.

18. Marshall T.W., Wallis M.K. (2013). Supermassive galactic centre with repulsive gravity. arXiv, 1303.5604 